\title{
Measurement of the Three-Dimensional Vibration Motion of the Ossicular Chain in the Living Gerbil
}

\author{
Willem F. Decraemer*, Ombeline de La Rochefoucauld ${ }^{\dagger}$, and \\ Elizabeth S. Olson ${ }^{\dagger}$ \\ ${ }^{*}$ Biomedical Physics, University of Antwerp \\ ${ }^{\dagger}$ Department of Otolaryngology, Head and Neck Surgery, Columbia University
}

\begin{abstract}
In previous studies 3D motion of the middle-ear ossicles in cat and human temporal bone were explored but models for hearing research has shifted in the last decades to smaller mammals and gerbil in particular has become a hearing model of first choice. In the present study we have measured with an optical interferometer the 3D motion of the malleus and incus in anesthetized gerbil for sound of moderate intensity ( $90 \mathrm{~dB}$ SPL) in a broad frequency range. To access the malleus and incus the pars flaccida was completely removed exposing the neck and head of the malleus and the incus from the malleus-incus joint to the long process of the incus and the plate of the lenticular process. In a previous study an approach through a hole in the bullar wall was used to study the stapes motion so that we now have a complete picture of the middle ear motion. In both approaches vibration measurements were done at 6 to 7 points per ossicle while the angle of observation was varied over approximately 30 degrees to enable calculation of the $3 \mathrm{D}$ velocity components. Knowledge of middle ear motion is of great importance in understanding how the middle ear transforms the acoustical input from the ear canal to the cochlea.
\end{abstract}

Keywords: gerbil, three-dimensional, vibration, middle-ear ossicles

PACS: 43.64.Ha

\section{INTRODUCTION}

In the last two decades the choice of mammals used for hearing experiments has moved away from cat to animals such as gerbil, guinea pig and mouse. Due to specific anatomical differences between the species and obvious differences in size this has as a consequence that experimental data that is amply available for cat cannot straightforwardly be used in the framework of the new animal model studies. Easy to breed and to house, and having an ear that is relatively large for the animal's overall body size, gerbil has now become a popular animal model. The size of the ossicles in gerbil is about half of that in cat, the ratio of the mass of the ossicles is hence about $1 / 8$ th. For middle ear mechanics research this means that even if we are still studying a three ossicle middle ear chain with similar gross anatomy we may expect that the dynamics of the system may be quite different. Knowledge of the specific dynamics of the gerbil middle ear is of great importance e.g. in cochlear studies to get to know how the acoustic input signal is transformed before reaching the cochlear fluid.

In a previous study we described how the motion of the stapes was measured through a posterior-inferior opening in the middle cavity wall [1]. In the present paper we completed the study of the ossicular chain motion by measuring the malleus and incus motion through the opening obtained by removing the pars flaccida. Both approaches allowed measurements on anesthetized animals. Note that measuring in vivo, it was not

(C) 2011 American Institute of Physics 978-0-7354-0975-0/\$30.00 
possible to measure the motion of the three ossicles in the same preparation.

\section{MATERIAL AND METHODS}

We will keep this section short as we can and refer to a previous study where we practiced a surgical preparation and experimental strategy almost identical to that used here [2].

Animal preparation. For measurement of the 3D motion of the malleus and incus experiments were performed on four gerbils, young animals of 50 to $70 \mathrm{~g}$. After sedation with ketamine they were deeply anesthetized with sodium pentobarbital. Supplemental doses were given when necessary. The body temperature was maintained at $37^{\circ} \mathrm{C}$ using an animal blanket. A tracheotomy was performed to maintain a clear airway. The care and use of animals were approved by the Institutional Animal Care and Use Committee of Columbia University.

Sound system and pressure measurement in the ear canal. Malleus and incus motion were measured in response to sound delivered in an open sound field configuration by an earphone directed to and placed nearby $(12-15 \mathrm{~mm})$ the ear canal, which was shortened up to its bony end part. The probe tip measured the sound near the edge of the earcanal [2]. We used pure tone stimuli that were stepped in frequency with SPLs kept constant at 80 or $90 \mathrm{~dB}$.

Vibration velocity measurement. For the velocity measurements we used a noncommercial heterodyne laser interferometer which is combined with a visual microscope [1]. The focused laser beam coincides with the center of the image of the microscope displayed on a video monitor; the microscope allows visualization of the object (malleus, incus) while the interferometer measures the vibration at the selected location.

The animal's body was supported and its head was immobilized by firmly clamping the head holder, which was cemented to the exposed top surface of the skull, to a post affixed to the positioning system. The positioning device consists of three orthogonal translation stages and two large, nested goniometers that are precisely aligned to rotate the object about the point of focus of the laser beam.

Vibration of the malleus and incus was measured through the opening obtained after removing the pars flaccida at a set of about seven to eight points on each ossicle, and this was repeated for five different observation angles. The ossicles are deeply anchored in the middle-ear cavity which restricted the change in observation angle of the vertical and horizontal goniometers to a range of about $-15^{\circ}$ to $+15^{\circ}$. During the rotation the animal, sound driver and probe microphone moved together as they were all mounted to the same object holder post of the positioning system keeping relative positions unchanged.

Rigid body velocities in an intrinsic malleus-incus reference frame. The coordinates of all observation points on a given ossicle and the amplitude and phase of the velocity at these points for all observation directions were used to calculate all three components of the rigid-body velocities which describe the motion as the translation of a reference point on the body and the rotation of the body about this point. Details of the mathematical procedure are given in [1]. 

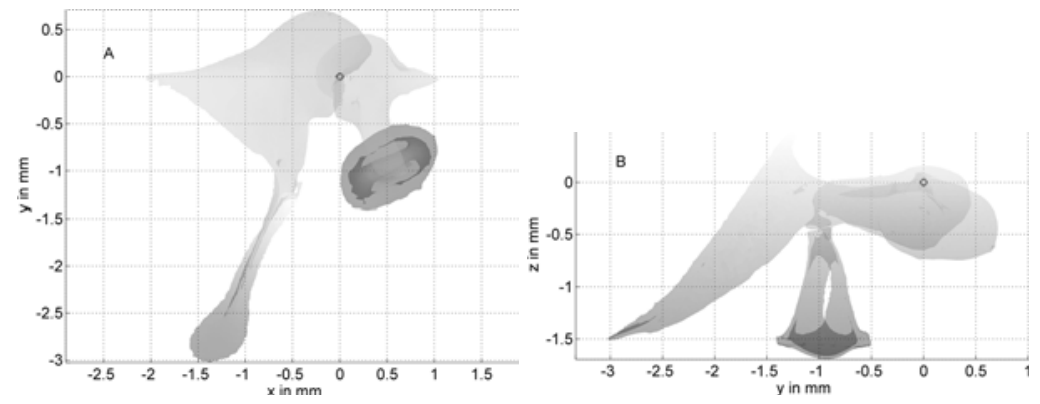

FIGURE 1. Two views of the ossicular chain in the intrinsic reference frame that we adopted for the malleus and the incus. The $x$-axis is directed from the anterior mallear tip to the posterior incudal process (panel A). The $z$-axis is along the stapes piston direction from the center of the footplate to the center of the stapes head (panel B). The origin indicated with an open circle, was chosen at the point where the $x$-axis crosses the malleus incus joint.

These velocity vectors are expressed in the coordinate system used during the experiment. In this reference system the object under study is quite arbitrarily oriented, which makes the interpretation of the frequency responses of the rotation and translation components almost impossible. In a second step these components are transformed in a meaningful intrinsic coordinate system for the malleus and incus: for gerbil it occurs that with the $x$-axis running from the anterior tip of the malleus to the posterior incudal process (let us call it the "anatomical rotation axis", the term "ligament axis" would require a ligament at the anterior mallear side which is not present in gerbil), we can align the $z$-axis nicely upward along the stapes piston direction (Fig. 1). We determine this transform in an intermediate step using a 3-D model of the ossicular chain constructed on the basis of a CT scan of a complete gerbil temporal bone [1]. This model is first registered in the position of the experiment. The coordinate transform that brings the ossicular chain model from the experimental into the intrinsic frame is then used to transform the translation and rotation vectors also in this reference frame.

\section{RESULTS}

In line with our previous papers the motion of the ossicles will be specified in terms of displacements rather than velocities, as it makes interpretation and visualization of the mode of vibration easier. Amplitudes and phases in the following sections are all expressed relative to the simultaneously recorded sound pressure.

\section{Translation displacements of the malleus and incus}

The translational displacements of the malleus and incus (Fig. 2) follow remarkably the same trend. At frequencies below $10 \mathrm{kHz}$ there is a major translation along the $y$ axis (practically the direction of the long process of the incus), and then it drops to a level more than 10 times smaller and comparable to that of the $x$ - and $z$-components. At frequencies up to $10 \mathrm{kHz}$ for the malleus and $15 \mathrm{kHz}$ for the incus all components are in phase, then phase differences are developed. 

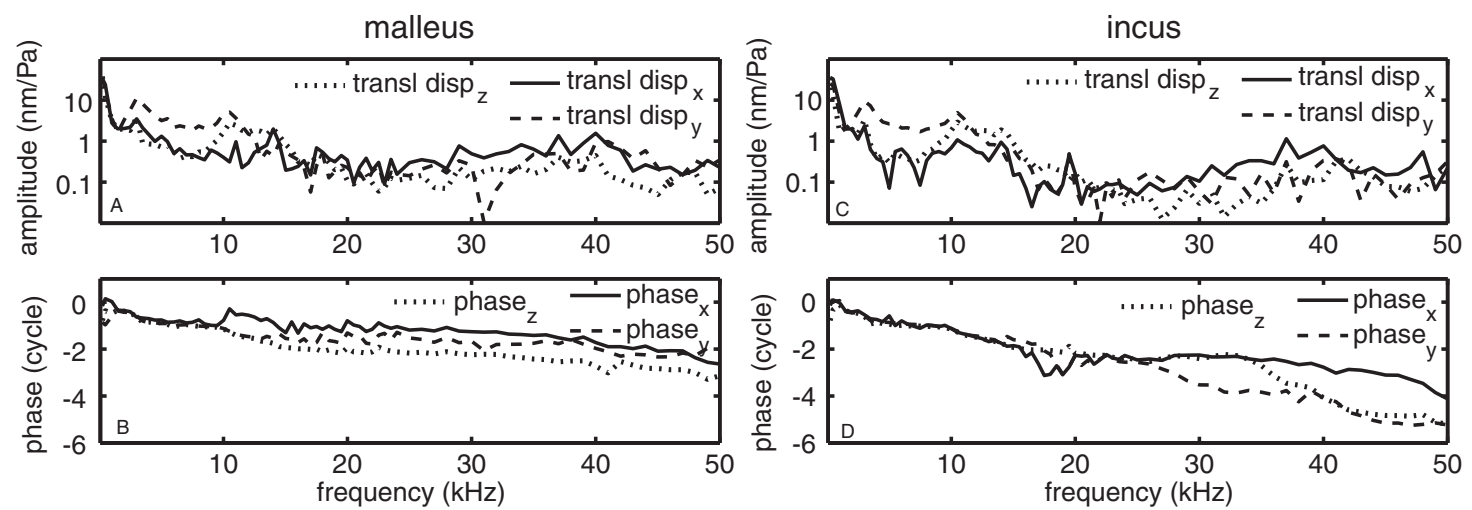

FIGURE 2. Translational amplitudes ( $\mathrm{A}$ and $\mathrm{C}$ ) and phases (B and D) of the malleus and incus vs frequency.
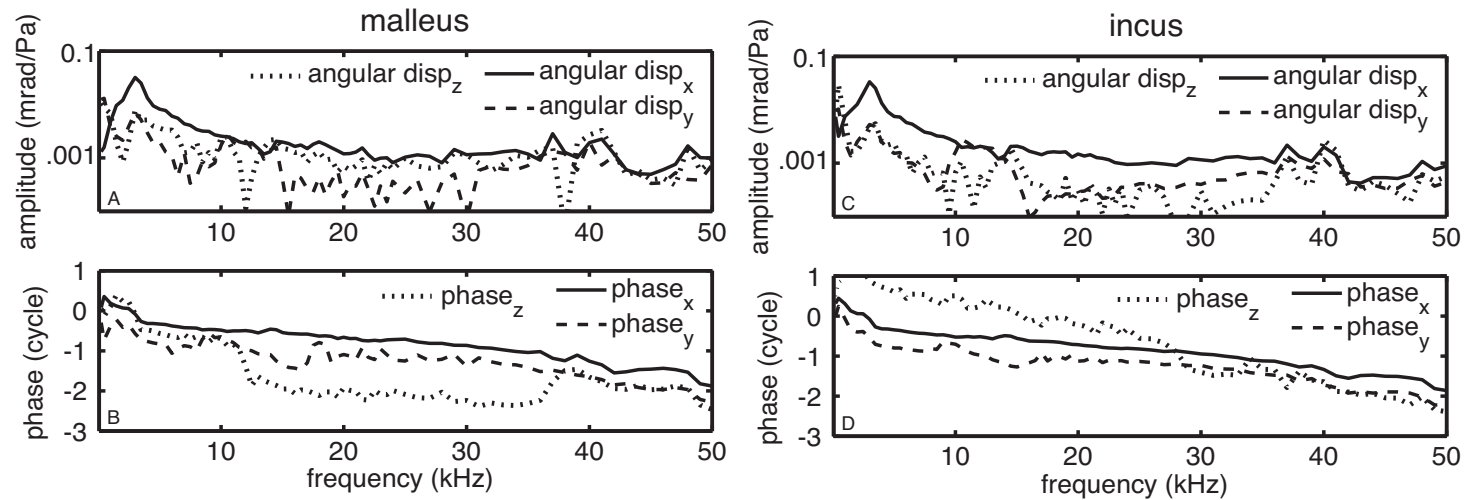

FIGURE 3. Rotational amplitudes ( $\mathrm{A}$ and $\mathrm{C}$ ) and phases ( $\mathrm{B}$ and $\mathrm{D})$ of the malleus and incus vs frequency.

\section{Rotation displacements of the malleus and incus}

The $\mathrm{x}$-component of the angular amplitudes for the malleus and incus are almost identical up to $40 \mathrm{kHz}$, and then there are small differences. With the choice of reference frame we anticipated to find the major angular displacement component along the $x$-axis. This is indeed verified by the results shown in Fig. 3. For the malleus, rotation about the $z$-axis becomes important as well above $10 \mathrm{kHz}$. For the incus the $y$-and $z$-components remain roughly 10 times smaller than the $x$-component, except at frequencies above 37 $\mathrm{kHz}$. The phases of the $x$-components for malleus and incus are almost alike throughout the entire frequency range with a nearly linear decreasing trend with frequency indicating a constant delay of about $60 \mu$ s with respect to the sound pressure. The $y$-component remains roughly out-of-phase with the $x$-component. For the malleus the phase of the $z$-component is also out-of-phase with the $x$-component between 12 and $35 \mathrm{kHz}$; for the incus the $z$-phase follows its own gradually, but more steeply decreasing trend. 

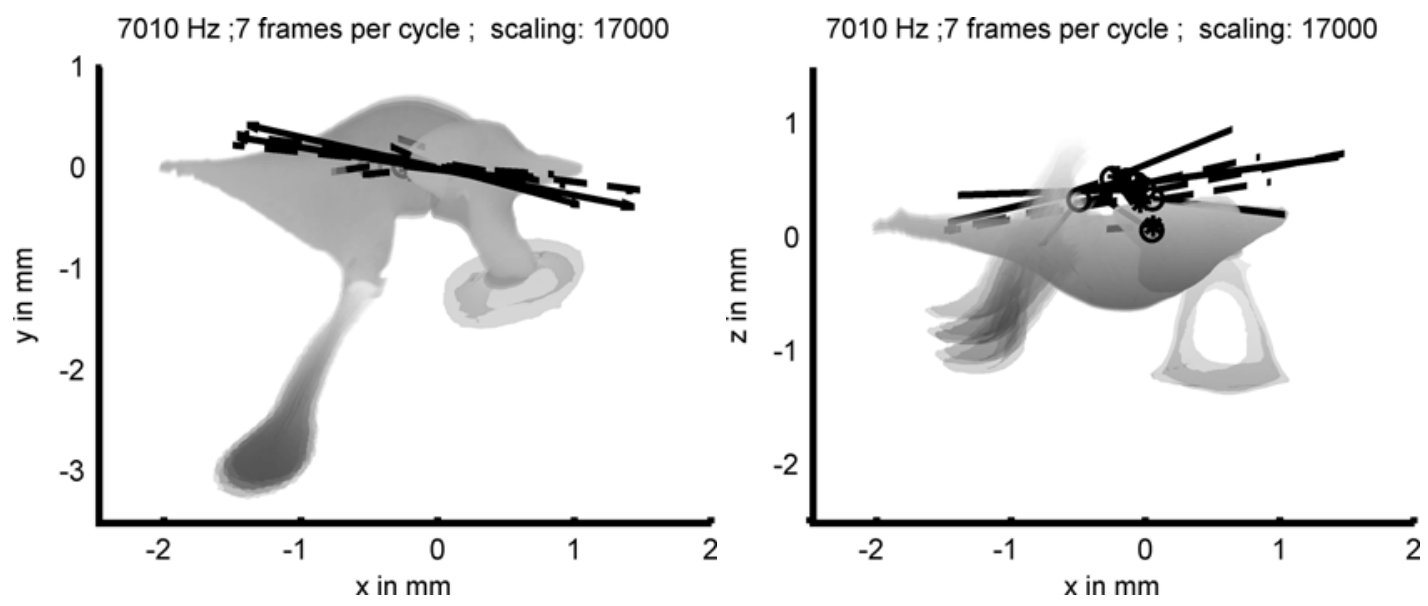

FIGURE 4. The malleus and incus are shown during their vibration motion at 7 equally spaced phases $(t=T \cdot[0,1, \ldots, 6] / 7)$ during the stimulus cycle. The rest positions of malleus and incus are also shown in a darker tone. Only for better orientation the rest position of the stapes is also shown. The frequency and scaling factor for the displacements are annotated at the top. Two views are shown: in left panel we look along the $z$-axis, in the right panel along the $y$-axis. For each phase in the cycle the screw axis is also shown (with a solid line for the malleus and a dashed line for the incus) originating from the point $R_{0}$ (the point on the screw axis closest to the origin $(0,0,0)$; dot for malleus, star for incus). For this relatively low frequency the rotation of the malleus takes place about an axis that is running slightly laterally to the malleus and incus block (right panel). The length of the screw axis vector was drawn to be proportional to the angular displacement at each instant of time; for maximal rotation angle the immediate rotation axis is making an angle of about $10^{\circ}$ with the anatomical axis ( $x$-axis, left panel) in the $x, y$-plane, and makes about the same angle with the $x$-axis in the $z, x$-plane (right). Note that the screw axes for malleus and incus are fairly well aligned at each phase of the motion.

\section{Mode of vibration of the malleus and incus}

To give a better visual idea of the mode of vibration we have calculated the screw axis, a mechanical concept that describes the motion of a rigid body as a rotation about an instantaneous rotation axis combined with a "slide" along this axis (this displacement is small here and is not discussed). Here we make no a priori assumptions about the location of the rotation axis with respect to the ossicles.

When compared with the classical hypothesis of middle ear vibration mode, the motion of the gerbil malleus and incus illustrated in Fig. 4 for a frequency of $7 \mathrm{kHz}$ (the results shown are typical for frequencies up to about $10 \mathrm{kHz}$ ) we see that the rotation axis is not fixed during the cycle. The shaded zone around the rest position in the left panel indicates rotation about the $z$-axis, in-plane motion of the manubrium. When the angular displacement is maximal, the direction of the screw axis is quite off from the anatomical axis. For the $x$-component of the angular velocity of the malleus and incusthis component defines the piston motion at the lenticular process - we found a nearly constant delay between the malleus and incus of about $3.6 \mu$ s, indicating that slippage between malleus and incus was small.

The middle ear ossicle motion is dependent on frequency as we will illustrate by showing in Fig. 5, in a similar way as we did in Fig. 4, results for a higher frequency, 15 

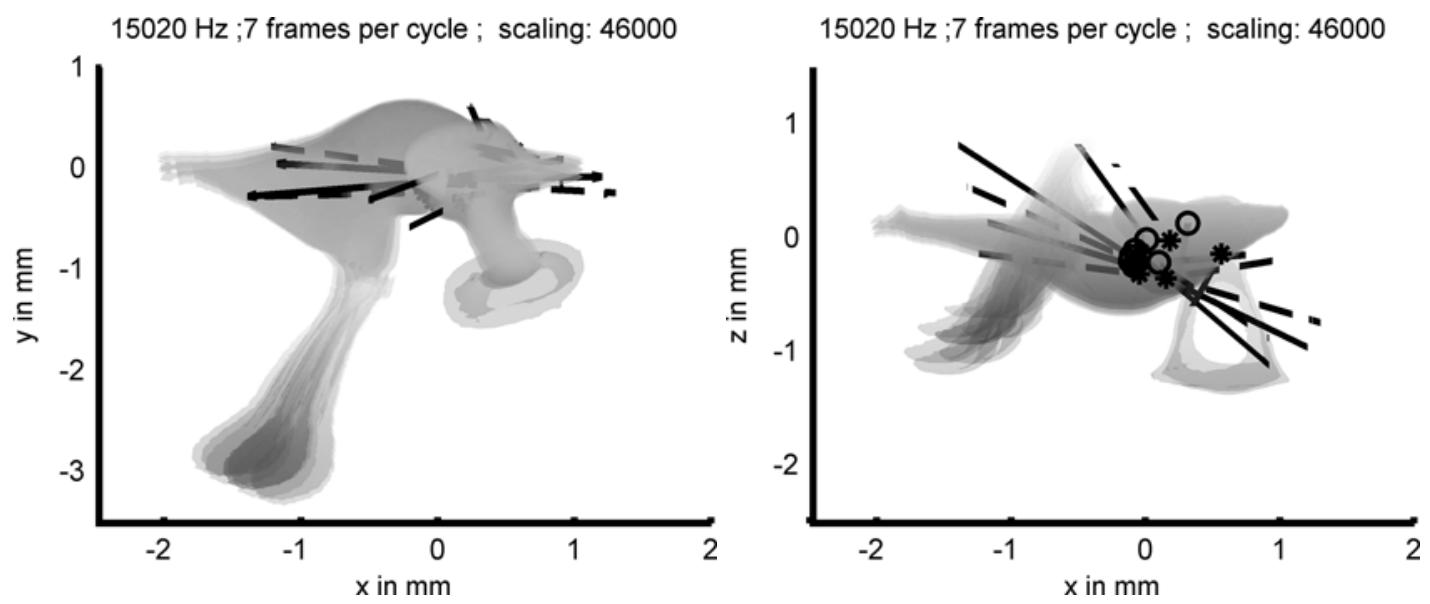

FIGURE 5. Figure similarly built up as Fig. 4, showing malleus and incus motion at a frequency of $15 \mathrm{kHz}$. Results are from the same experiment as those shown in Fig. 4.

$\mathrm{kHz}$. With increasing frequency changes in vibration mode occur. At the frequency of $15 \mathrm{kHz}$, Fig. 5 shows that the mode of vibration has changed significantly from that at $7 \mathrm{kHz}$ : the rotation axis is wobbling about the $z$-axis (left panel) and its position in the $z, x$-plane is quite different too.

\section{DISCUSSION AND CONCLUSIONS}

Results for other gerbils showed that inter-animal differences were present but up to 10 $\mathrm{kHz}$ the mode of vibration was quite similar for all our animals, at higher frequencies differences were more pronounced.

The mode of vibration of the gerbil middle ear is quite different from that in cat and human that we studied earlier. In gerbil the mode changes with frequency are less pronounced and slippage in the malleus-incus joint was smaller. The considerably smaller mass of the gerbil ossicles is most probably the reason for it. Our findings are useful in determining the sound transfer characteristics through the middle ear into the cochlea and may serve as a reference for validation of mathematical middle ear models.

\section{ACKNOWLEDGMENTS}

This work was supported by NIH/NICDC grant DC003130, the Emil Capita Fund, and the Fund for Scientific Research (Flanders, Belgium).

\section{REFERENCES}

[1] Decraemer WF, de La Rochefoucauld O, Dong W, Khanna SM, Olson ES (2007) Scala vestibuli pressure and three-dimensional stapes velocity measured in direct succession in gerbil. J Acoust Soc Am 121:2774-2791

[2] de La Rochefoucauld O, Decraemer WF, Khanna SM, Olson ES (2008) Simultaneous measurements of ossicular velocity and intracochlear pressure leading to the cochlear input impedance in gerbil. $\mathrm{J}$ Assoc Res Otolaryngol 9:161-177 
Copyright of AIP Conference Proceedings is the property of American Institute of Physics and its content may not be copied or emailed to multiple sites or posted to a listserv without the copyright holder's express written permission. However, users may print, download, or email articles for individual use. 